\title{
Three-Dimensionally and Stackable C-Axis-Aligned Crystalline Indium-Gallium-Zinc Oxide Field-Effect Transistor with Gate Length of 6.8-nm
}

Hitoshi Kunitake ( $\square$ hk1372@sel.co.jp)

Semiconductor Energy Laboratory Co., Ltd. https://orcid.org/0000-0003-1187-4590

Kazuki Tsuda

Semiconductor Energy Laboratory Co., Ltd.

\section{Satoru Saito}

Semiconductor Energy Laboratory Co., Ltd.

\section{Naoki Okuno}

Semiconductor Energy Laboratory Co., Ltd.

Masahiro Takahashi

Semiconductor Energy Laboratory Co., Ltd.

\section{Masashi Oota}

Semiconductor Energy Laboratory Co., Ltd.

\section{Toshiki Hamada}

Semiconductor Energy Laboratory Co., Ltd.

Yoshinobu Asami

Semiconductor Energy Laboratory Co., Ltd.

Hiromi Sawai

Semiconductor Energy Laboratory Co., Ltd.

\section{Motomu Kurata}

Semiconductor Energy Laboratory Co., Ltd.

\section{Toshiya Endo}

Semiconductor Energy Laboratory Co., Ltd.

\section{Kentaro Sugaya}

Semiconductor Energy Laboratory Co., Ltd.

\section{Masaru Nakano}

Semiconductor Energy Laboratory Co., Ltd.

Ryota Hodo

Semiconductor Energy Laboratory Co., Ltd.

\section{Shinya Sasagawa}

Semiconductor Energy Laboratory Co., Ltd.

\section{Toshikazu Ono}


Semiconductor Energy Laboratory Co., Ltd.

\section{Tsutomu Murakawa}

Semiconductor Energy Laboratory Co., Ltd.

\section{Masahiro Wakuda}

Semiconductor Energy Laboratory Co., Ltd.

\section{Shunpei Yamazaki}

Semiconductor Energy Laboratory Co., Ltd.

\section{Article}

Keywords:

Posted Date: January 19th, 2022

DOI: https://doi.org/10.21203/rs.3.rs-1215280/v1

License: (c) (i) This work is licensed under a Creative Commons Attribution 4.0 International License. Read Full License 


\title{
Three-Dimensionally and Stackable $C$-Axis-Aligned Crystalline Indium-Gallium-Zinc Oxide Field-Effect Transistor with Gate Length of $6.8 \mathrm{~nm}$
}

\author{
H. Kunitake, K. Tsuda, S. Saito, N. Okuno, M. Takahashi, M. Oota, T. Hamada, Y. \\ Asami, H. Sawai, M. Kurata, T. Endo, K, Sugaya, M. Nakano, R. Hodo, S. Sasagawa, \\ T. Ono, T. Murakawa, M. Wakuda, and S. Yamazaki
}

2 Abstract- Hardware is required to be further miniaturized aiming at advancement of the Internet of things and artificial intelligence. Widely used $\mathrm{Si}$ transistors, which have 6 achieved miniaturization on the order of $10 \mathrm{~nm}$, are apparently difficult to further 8 miniaturize, and stacking techniques have been developed as a breakthrough. Our 10 IGZO FETs have a gate length of $6.8 \mathrm{~nm}$ or 11 less owing to the wide band gap of IGZO and an optimized transistor structure, and can be highly integrated by a contact formation technique.

\section{INTRODUCTION}

In the history of advancement of complementary-metal-oxide-semiconductor (CMOS) technologies, conventional miniaturized planar field-effect transistors (FETs), especially $32 / 28 \mathrm{~nm}$ node devices and smaller ones, have already reached the limits of accuracy of patterning, and many studies have examined FETs having threedimensional (3D) gate structures such as Fin FETs and gate-all-around (GAA) transistors, rather than planar FETs [1-2] (Fig. 1). For higher integration, higher performance, and lower power consumption, techniques for three-dimensionally stacking FETs have been actively studied. In such 3D stacking techniques, including monolithic stacking and bonding, reducing the footprint of devices formed in each layer is important for improving the degree of integration; accordingly, the technology node and the degree of integration of the devices fabricated in a back-end-of-line (BEOL)

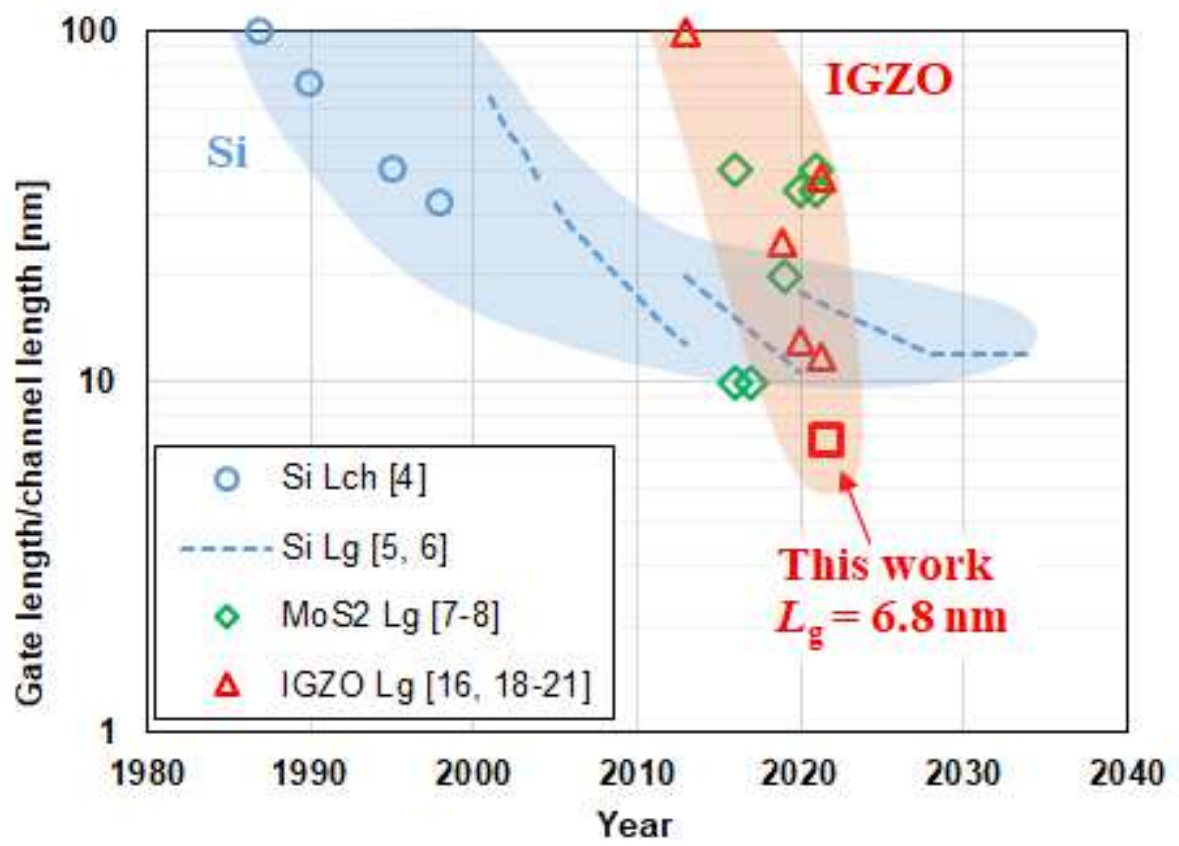

Fig. 1 | Changes in channel length $\left(L_{c h}\right)$ or gate length $\left(L_{g}\right)$ in SiFET, MoS 2 FET, and IGZO FET 
process are expected to be as close to those of the most advanced $\mathrm{Si}$ CMOS circuit as possible. As materials and techniques for stacking FETs over a CMOS circuit, carbon nanotubes, two-dimensional materials, polycrystalline $\mathrm{Si}$, and bonding have been proposed, for example [3]. However, a technique which fulfills all the requirements, including a low-temperature process applicable to a BEOL process, a technology node close to that of a Si CMOS circuit [56], excellent on/off characteristics, and lower power consumption, is under development.

As one response to these technical needs, Indium-gallium-zinc oxide (IGZO), have been attracting attention. IGZO typically has a wider band gap $\left(E_{g}>3 \mathrm{eV}\right)$ than $\mathrm{Si}$ $\left(E_{g}=1.1 \mathrm{eV}\right)$, and is a promising material for enabling low-power devices which cannot be obtained only with Si. IGZO has been often used mainly for ultra-low-power displays [911]. Because IGZO FET can be stacked monolithically over a Si CMOS circuit by a low-temperature process $\left(<450^{\circ} \mathrm{C}\right)$, various applications of IGZO as a channel material for low-power devices have been reported [12-17]. Of several types of IGZO, $c$-axisaligned-crystalline IGZO (CAAC-IGZO) which has high crystallinity only in the $c$-axis direction has been getting attention because of its unique crystal structure and high thermal and structural stability as well as ease of sputter deposition [18-19]. In recent years, miniaturization of IGZO FETs has also been actively studied with their application to LSI in view [20-21]. However, when the thickness of a gate insulating film (GI) is reduced according to the scaling law along with miniaturization of the FET, GI leakage might increase to make the IGZO FET compromise its advantage, the low power consumption [22-23]. Furthermore, the carrier concentrations in source, channel, and drain regions are required to be adequately controlled in short channel FETs [24]; however, $\mathrm{V}_{\mathrm{OH}} \mathrm{H}$, which is a defect generated by bonding of remaining hydrogen to an oxygen vacancy ( $\left.V_{0}\right)$ to serve as a donor source in an IGZO film [25], is diffused to react in IGZO by thermal budget. Thus, in short channel FETs, a short circuit easily occurs in an $\mathrm{n}^{+}$region between the source and drain electrodes, which hinders achieving adequate on/off characteristics. It is accordingly important to develop a devised process capable of controlling distribution of $V_{0}$ and hydrogen so that short channel FETs can be fabricated using IGZO in their channel layers. In this article, we fabricated a miniaturized IGZO FET having a trench-gate-self-aligned (TGSA) structure by a low-temperature process $\left(<450^{\circ} \mathrm{C}\right)$ [26-27]. Cross-sectional scanning transmission electron microscope (STEM) observation showed that the fabricated CAAC-IGZO FET had a gate electrode length $\left(L_{g}\right)$ of $6.8 \mathrm{~nm}$ and a channel width $(W)$ of $32.1 \mathrm{~nm}$. As a fabrication process of CAAC-IGZO FETs, we developed a process in which oxygen is selectively supplied to only the channel region to form a junctionless transistor having $\mathrm{n}^{+} / \mathrm{n}^{-} / \mathrm{n}^{+}$ regions between the source and drain electrodes. The amount of carriers accumulated in the channel region can be controlled by a top gate electrode, which covers a channel side wall as in a Fin FET. IGZO FETs are of an accumulation type and thus have a small characteristics length between the source/drain and the channel [28]. As a result, when including IGZO, even a junctionless, short channel FET in which the Fermi level only slightly differs between the source/drain and the channel can more easily achieve a high on/off ratio than inversion SiFETs. A bottom gate electrode is provided under the channel separately from the top gate electrode in our FET; thus, the threshold voltage ( $V_{\text {th }}$ ) can be adjusted to a desired value by changing the bottom gate voltage ( $\left.V_{b s}\right)$. The IGZO FETs having these features are less likely to be affected by short channel effects [29] and thus our process should be the key to contributing to further miniaturization. 


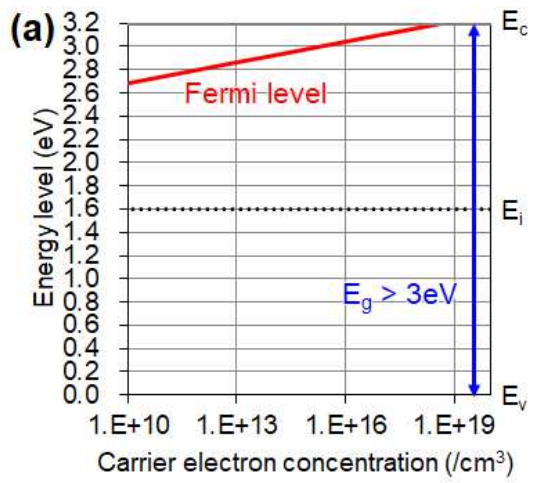

Schematic diagram of CAAC-IGZO FET

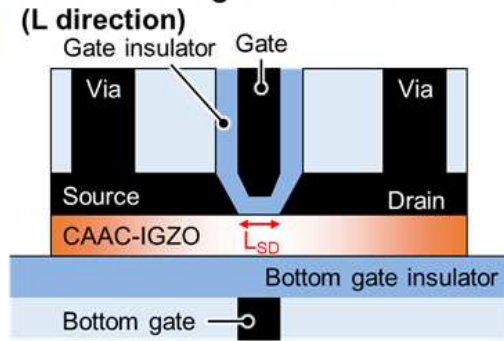

$E_{c}$ band offset(L direction)
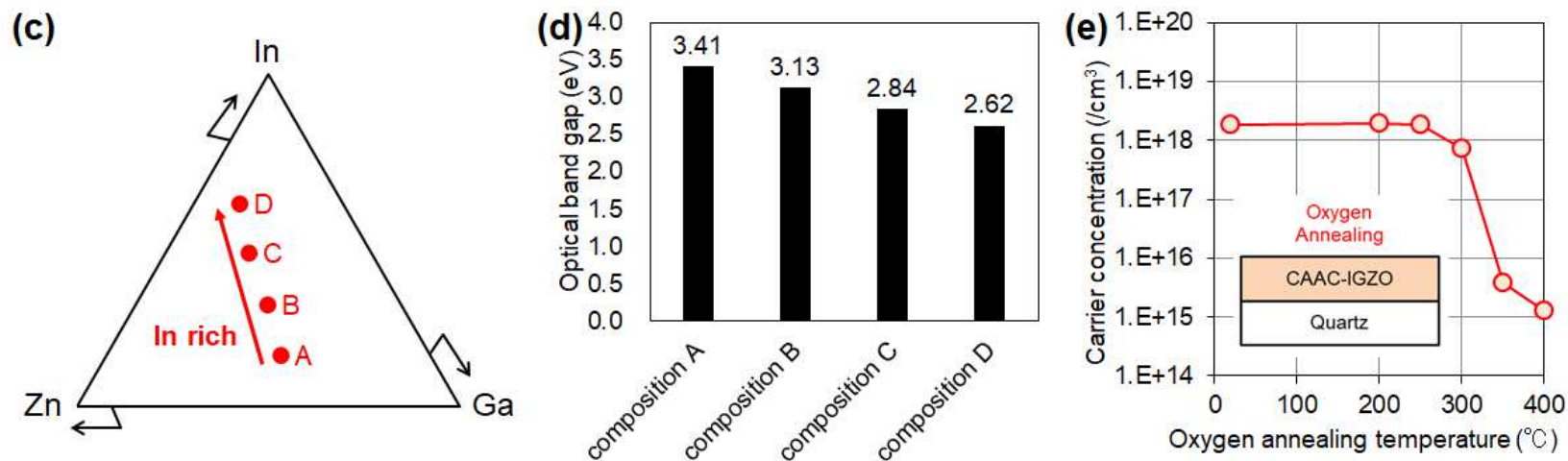

Fig. 2 | Data on various fundamental physical properties of IGZO. (a) Relation between the carrier concentration and the Fermi level, which was calculated from $\mathrm{n}=N_{\mathrm{c}} \exp \left(\left(E_{\mathrm{f}}-E_{\mathrm{c}}\right) / \mathrm{k}_{\mathrm{B}} T\right)$ where $\mathrm{n}$ represents the carrier concentration, $N_{c}$ represents the density of state in the conduction band (set to $5 \times 10^{18} / \mathrm{cm}^{3}$ ), $E_{\mathrm{f}}$ represents the Fermi level, $E_{\mathrm{c}}$ represents the energy at the conduction band minimum, $\mathrm{k}_{\mathrm{B}}$ represents the Boltzmann constant, and $T$ represents absolute temperature (set to $300 \mathrm{~K}$ ). (b) A schematic cross-sectional diagram of an CAAC-IGZO FET in the $L_{c h}$ direction and a schematic band offset diagram of the conduction band in the same direction. (c) A phase diagram of IGZO. A, B, C, and D are in order of increasing In richness. (d) Optical band gaps of IGZO with the compositions A, $\mathrm{B}, \mathrm{C}$, and D. (e) Carrier concentrations in CAAC-IGZO thin films after annealing in an oxygen atmosphere. The carrier concentration of the CAAC-IGZO films had been increased by Vo and VoH formation.

\section{Physical Properties of Crystalline igzo} Despite being oxides, IGZO, ZnO, and other materials have the properties of semiconductors. The carrier type of many kinds of oxide semiconductor is an n-type. Although p-type oxide semiconductor has also been studied, their performance is inferior to n-type oxide semiconductor [30].

Device simulation results of IGZO FETs have already revealed that FET characteristics with a high on/off ratio can be obtained when the donor concentration in a channel region of an IGZO film is kept at approximately $10^{16} / \mathrm{cm}^{3}$ to $10^{17} / \mathrm{cm}^{3}$ or lower [31]. For example, the Fermi level $\left(E_{c}-\right.$ $\left.E_{f}\right)$ when the carrier concentration is $10^{16} / \mathrm{cm}^{3}$ is approximately $0.2 \mathrm{eV}$, indicating $\mathrm{n}^{-}$-type conductivity. In a short channel FET, even in the case where the channel region
153 has a low carrier concentration, the 154 conduction band minimum of the channel 155 region is lowered by the conduction band 156 lowering effect, so that a conduction band 157 offset between the source/drain region and 158 the channel region is reduced [32]. 159 Accordingly, an FET including IGZO can be 160 regarded as a junctionless accumulation 161 transistor with an $\mathrm{n}^{+} / \mathrm{n}^{-} / \mathrm{n}^{+}$structure in which 162 the channel region is of an $\mathrm{n}^{-}$type and the 163 source and drain regions are of an $\mathrm{n}^{+}$type 164 (Figs. 2(a) and 2(b)).

165 The band gap of IGZO is slightly 166 dependent on its composition, i.e., In: Ga: Zn 167 ratio, but is approximately larger than $3 \mathrm{eV}$ 168 when the In: Ga: $\mathrm{Zn}$ ratio is around 1:1:1 or 169 the $\mathrm{Ga}$ ratio is higher than that in the 170 composition In: $\mathrm{Ga}: \mathrm{Zn}=1: 1: 1$ 171 (Figs. 2(c) and 2(d)). Because of such a 172 wide band gap, an CAAC-IGZO FET has a 
high breakdown voltage and other features, a notable one of which is an extremely low off-state current. Typical FETs can have an increased current amount by having an increased $W$, whereas CAAC-IGZO FETs cannot have an off-state current above the lower detection limit of a usual parameter analyzer even when the $W$ is extremely large. In some reports, a circuit dedicated for measuring the low off-state current was fabricated to reveal an extremely low offstate current of an CAAC-IGZO FET [33]. Such low-off-state-current characteristics have been observed in miniaturized CAACIGZO FETs as well [34]. By including FETs with the above feature, memory devices can retain data for a long time.

As an origin of donor states in IGZO, $\mathrm{V}_{\mathrm{OH}}$ in which hydrogen is bonded to $V_{0}$ has been reported [25]. Oxygen and hydrogen are respectively a constituent element and a ubiquitous element and are thus very hard to control. Accordingly, controlling the amounts of hydrogen and oxygen around IGZO is of importance. By contrast, a metal in contact with IGZO needs to avoid getting oxidized [24]. Blocking hydrogen by surrounding an CAAC-IGZO FET with $\mathrm{SiN}_{x}$ or the like has been reported to increase the reliability [35]. In an FET fabrication process, $\mathrm{V}_{\mathrm{O}}$ and $\mathrm{V}_{\mathrm{O}} \mathrm{H}$ are inevitably formed in IGZO of the channel region to increase the carrier concentration. In such a case, supplying oxygen to IGZO of the channel region can cure $\mathrm{Vo}_{\mathrm{o}}$ and $\mathrm{VoH}$ to reduce the carrier concentration. Fig. 2(e) shows experimental results plainly indicating that annealing in an oxygen atmosphere can reduce the carrier concentration of an IGZO thin film which has been increased by $V_{O}$ and $\mathrm{VoH}$ formation. Since controlling the carrier concentration in the channel region by oxygen annealing is not practical in a miniaturized CAAC-IGZO FET, we have developed a structure and a method in which oxygen is selectively supplied to only the channel region to reduce the carrier concentration in the channel region to an adequate level.

\section{Fin Structure with Bottom Gate} In this study, we fabricated a CAAC-IGZO FET having a bottom gate electrode by a TGSA gate last process (Fig. 3(a)) [26-27]. The CAAC-IGZO FET had an $L_{g}$ of $6.8 \mathrm{~nm}$. As a channel material of the FET, 15-nmthick CAAC-IGZO with $c$-axis aligned crystallinity was formed. A buffer layer was formed under CAAC-IGZO to block diffusion of oxygen from a bottom gate insulating film (BGl). As the source and drain electrodes, $\mathrm{TaN}_{x}$ was deposed by a sputtering method. $\mathrm{TaN}_{x}$ has a higher compressive stress than CAAC-IGZO [24]. This compressive stress causes distortion in CAAC-IGZO and increases the lattice constant. The increase in lattice constant generates $V_{0}$ in CAACIGZO. Hydrogen enters $\mathrm{Vo}_{0}$ to form $\mathrm{VoH}$, so that the region under $\mathrm{TaN}_{x}$ has n-type conductivity. $\mathrm{SiN}_{x}$ as a barrier film was formed around the FET to block entry of hydrogen. Furthermore, a $\mathrm{HfO}_{x}$ film absorbing hydrogen was provided inward from the hydrogen barrier film [35]. By inhibiting hydrogen diffusion through the above process, $\mathrm{VoH}$ formation in the channel region was suppressed. To eliminate $V_{0}, V_{0}$ must be terminated by oxygen. Thus, a sputter-deposited $\mathrm{AlO}_{x}$ film was provided over the gate. Oxygen can be diffused from the $\mathrm{AlO}_{x}$ film to CAAC-IGZO. The amount of oxygen can be controlled by adjusting the deposition conditions of the $\mathrm{AlO}_{x}$ film.

Through the above process, the donor concentration was successfully varied between the channel region and the source and drain regions in the FET. In our CAACIGZO FET, the electric field therearound can be blocked with the bottom gate electrode as in a GAA structure. It is to be noted that the bottom gate electrode of our FET works independently of the top gate electrode unlike in a typical GAA structure in which the bottom gate electrode works as a usual gate electrode. The bottom gate electrode working independently can shift the $V_{\text {th }}$ of the FET, and owing to this feature, the FET can be used for a $V_{\text {th }}$ correction circuit, for example [36]. Our CAAC-IGZO FET has a 


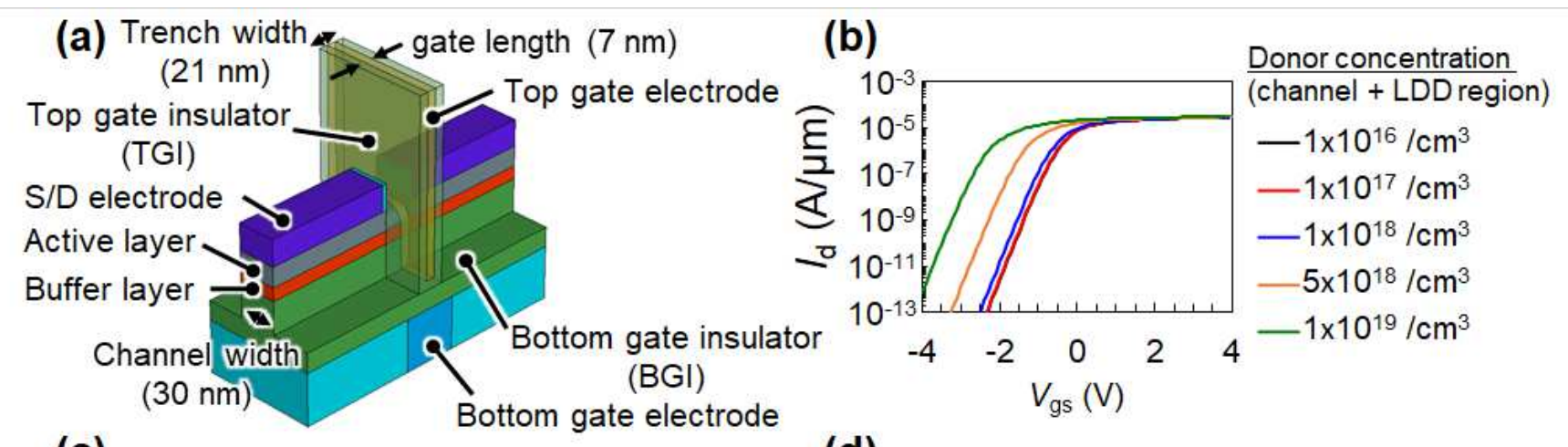

(c)

(d)
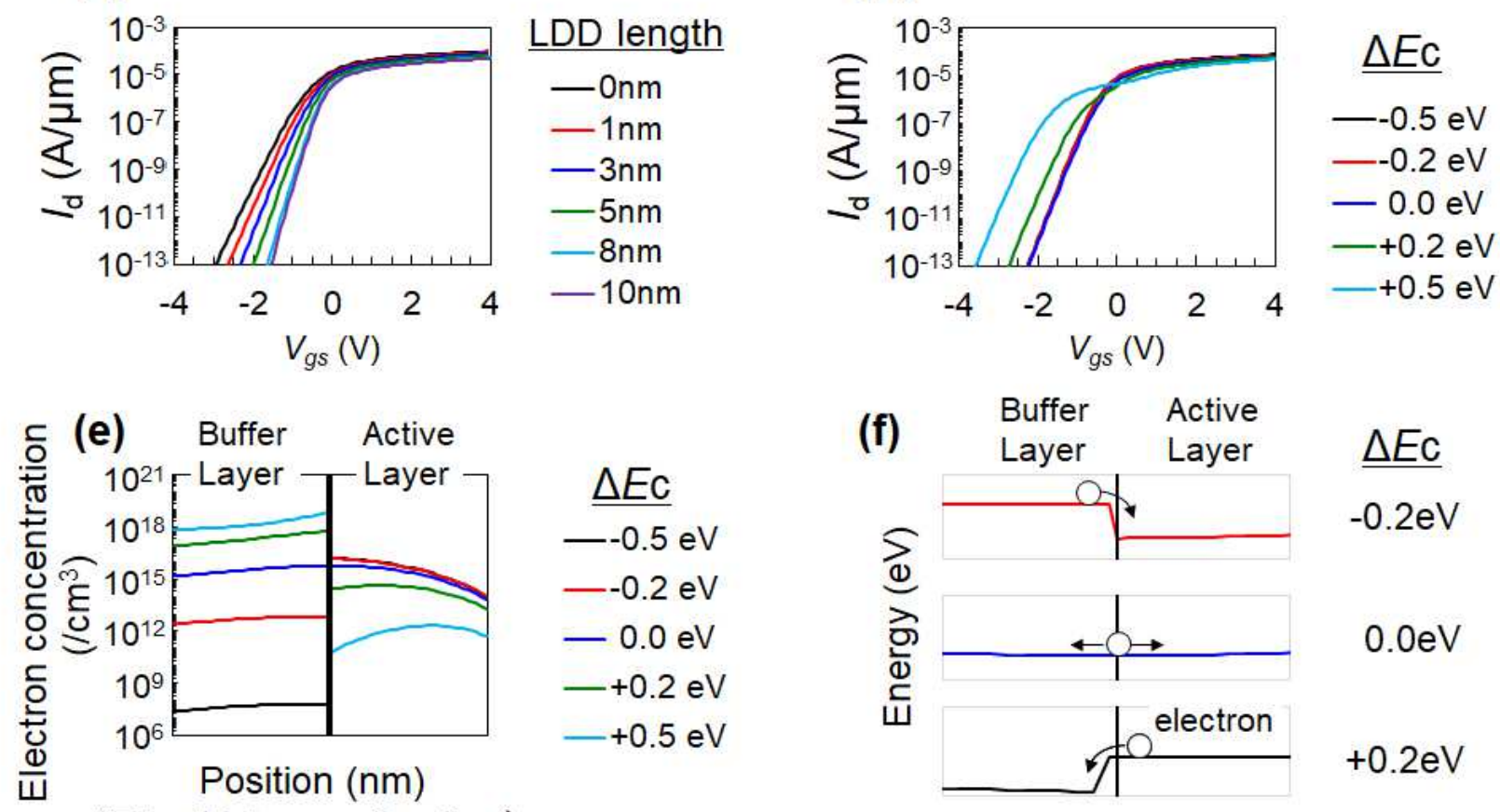

(Film thickness direction)

\begin{tabular}{|l|}
\hline Difference in electron affinity $(\Delta \mathrm{Ec})$ \\
(buffer layer) - (active layer)
\end{tabular}

(f)

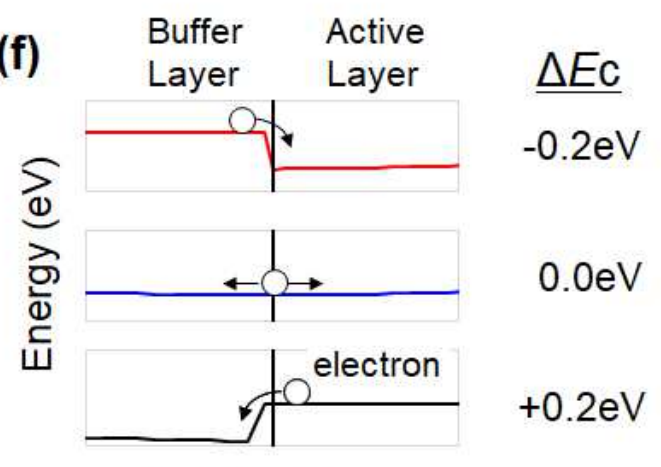

Position (nm)

(Film thickness direction)

Fig. 3 | Comparison of device simulation results with various parameters. (a) A perspective view of a Fin FET with a bottom gate. (b) $l_{\mathrm{d}}-V_{\mathrm{gs}}$ characteristics with varied donor concentrations of the channel and LDD regions $\left(V_{\mathrm{ds}}=0.1 \mathrm{~V}\right.$, source voltage $\left.\left(V_{s}\right)=V_{b s}=0 \mathrm{~V}\right)$. (c) $l_{d}-V_{g s}$ characteristics with varied LDD lengths $\left(V_{d s}=0.1 \mathrm{~V}, V_{b s}=0 \mathrm{~V}\right)$. (d) $l_{d}-V_{g s}$ characteristics with varied differences in electron affinity $\left(V_{\mathrm{ds}}=0.1 \mathrm{~V}, V_{\mathrm{bs}}=0 \mathrm{~V}\right)$. (e) Electron concentrations with varied differences in electron affinity (at the center of the channel) $\left(V_{\mathrm{ds}}=0.1 \mathrm{~V}, V_{\mathrm{gs}}=-1 \mathrm{~V}, V_{\mathrm{bs}}=0 \mathrm{~V}\right)$. (f) Energies at the conduction band minimum with varied differences in electron affinity (at the center of the channel) $\left(V_{\mathrm{ds}}=0.1 \mathrm{~V}\right.$, $\left.V_{\mathrm{gs}}=-1 \mathrm{~V}, V_{\mathrm{bs}}=0 \mathrm{~V}\right)$. current. The top gate insulating film (TGI) has a physical thickness of $7 \mathrm{~nm}$ (equivalent oxide thickness, or EOT: $5.1 \mathrm{~nm}$ ) and the BGI has a physical thickness of $40 \mathrm{~nm}$ (EOT: $25 \mathrm{~nm}$ ). Such $\mathrm{Gl}$ thicknesses are much larger than those of miniaturized FETs of the same size used in SiLSI [37]. This can inhibit gate leakage current despite application of voltage as high as several volts. Conversely,

a large Gl thickness means weakened control by a gate electric field, which makes it difficult to inhibit the influence of drain induced barrier lowering (DIBL) in a short channel FET. In this study, we attempted fabrication of a miniaturized CAAC-IGZO FET having high current drive capability. In a CAAC-IGZO FET, the influence of impurity scattering is not dominant and thus, the mobility can be increased by increasing the 
donor concentration [38]. Therefore, an effective method of enhancing current drive capability is to slightly increase the carrier concentration of the channel region. However, an increase in carrier concentration causes the Fermi level to get close to the conduction band, in which case the $V_{\text {th }}$ of a CAAC-IGZO FET is easily negatively shifted and the on/off ratio decreases (Fig. 2(a)). Thus, measures must be taken to obtain a high on/off ratio. In particular, relaxing the drain electric field and enhancing the controllability by the gate electrode are important in a short channel FET. We examined an effective way to refine the process, using device simulation.

First, a method for relaxing a drain electric field was examined. We can vary the donor concentration between regions through well controlled dehydrogenation and oxygen adding treatment. It is thus possible to form a lightly doped drain (LDD) region in order to suppress the drain electric field. Fig. 3(b) shows comparison of drain current $\left(l_{d}\right)$-gate voltage $\left(V_{\mathrm{gs}}\right)$ characteristics of FETs having an LDD length of $3 \mathrm{~nm}$ and varied donor concentrations. By reducing the donor concentration in the channel and LDD regions, a negative shift in $V_{\text {th }}$ can be suppressed. However, the $V_{\text {th }}$ ceased to positively shift at approximately $10^{17} / \mathrm{cm}^{3}$; thus, to further improve the characteristics, a measure other than reducing the donor concentration is needed. Fig. 3(c) shows comparison of $l_{d}-V_{g s}$ characteristics of FETs having a donor concentration in the channel and LDD regions of $10^{16} / \mathrm{cm}^{3}$ and varied LDD lengths. As can be seen in the graph, a longer LDD length led to a better subthreshold swing (SS) and resultantly to a positive shift in $V_{\text {th. }}$. This is because the presence of the LDD region relaxes the drain electric field. Nevertheless, normally-off characteristics were not obtained only by taking these measures.

Next, a method for enhancing the controllability by the gate electrode was examined. A typical example of a method for effectively enhancing the controllability by the gate electrode is reducing the
340 thicknesses of the TGI and active layer. 341 However, a reduction in the thickness of the $342 \mathrm{TGI}$ is not preferred because it might 343 increase gate leakage current, contradictory 344 to our aim of minimizing off-state leakage 345 current. Besides, a reduction in thickness of 346 the active layer is not preferred either 347 because it reduces current drive capability, 348 contradictory to our aim of enhancing it. 349 Accordingly, we examined methods other 350 than reducing the thicknesses of the TGI and 351 active layer. To control the oxygen adding 352 process, we form, under CAAC-IGZO, the 353 buffer layer blocking oxygen diffusion from 354 the BGI [24]. Because this buffer layer is 355 inevitable for controlling oxygen diffusion 356 under the status quo, we decided to examine 357 methods in which this buffer layer is utilized 358 to increase the controllability by the top gate 359 electrode.

360 In the first method, a difference in electron 361 affinity $\left(\Delta E_{\mathrm{c}}\right)$ between the buffer layer and 362 active layer was utilized. Fig. 3(d) shows 363 comparison of $l_{\mathrm{d}}-V_{\mathrm{gs}}$ characteristics with 364 varied $\Delta E_{\mathrm{c}}$. Here, $\Delta E_{\mathrm{c}}$ is defined as a value 365 obtained by subtracting the electron affinity 366 of the active layer from that of the buffer layer. 367 When the buffer layer had higher electron 368 affinity $\left(\Delta E_{\mathrm{c}}>0 \mathrm{eV}\right)$, the FET characteristics 369 curves exhibited a negative shift and 370 included a hump. Fig. 3(e) shows 371 comparison of electron concentration 372 distribution when the $V_{\mathrm{gs}}$ was $-1 \mathrm{~V}$ and the 373 drain voltage $(V \mathrm{ds})$ was $0.1 \mathrm{~V}$. In the case 374 where the buffer layer had higher electron 375 affinity $\left(\Delta E_{\mathrm{c}}>0 \mathrm{eV}\right)$, the electron 376 concentration of the buffer layer was higher 377 than that of the active layer. This is because 378 electrons will easily move to a layer with 379 higher electron affinity in the case of 380 heterojunction (Fig. 3(f)). Therefore, 381 electrons can be efficiently moved to the 382 active layer by making the electron affinity of 383 the buffer layer lower than that of the active 384 layer $\left(\Delta E_{\mathrm{c}}<0 \mathrm{eV}\right)$. In this way, only the 385 active layer in IGZO needs to be subjected 386 to the carrier control by the top gate electric 387 field, so that the controllability by the top gate 388 can be increased. In this state, moreover, an 
(a)

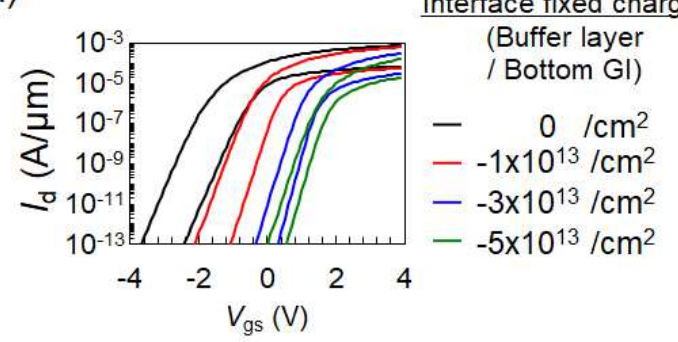

(c)

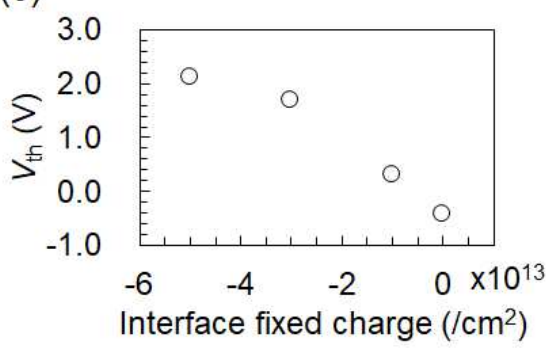

(b)

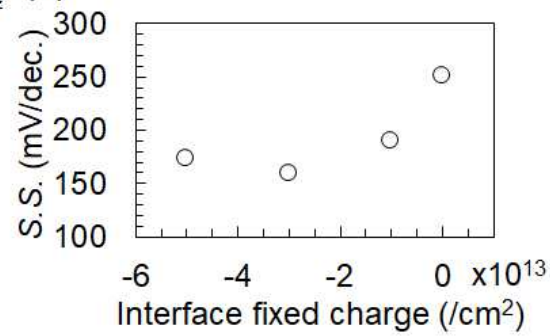

(d) $\quad$ IBL $=\frac{V_{t h}\left(V_{d s}=0.1 V\right)-V_{t h}\left(V_{d s}=1.2 V\right)}{\Delta V}$

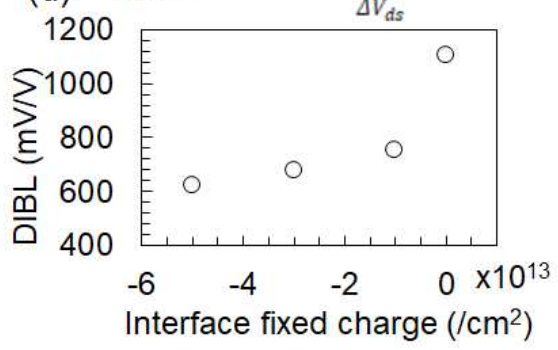

(e)

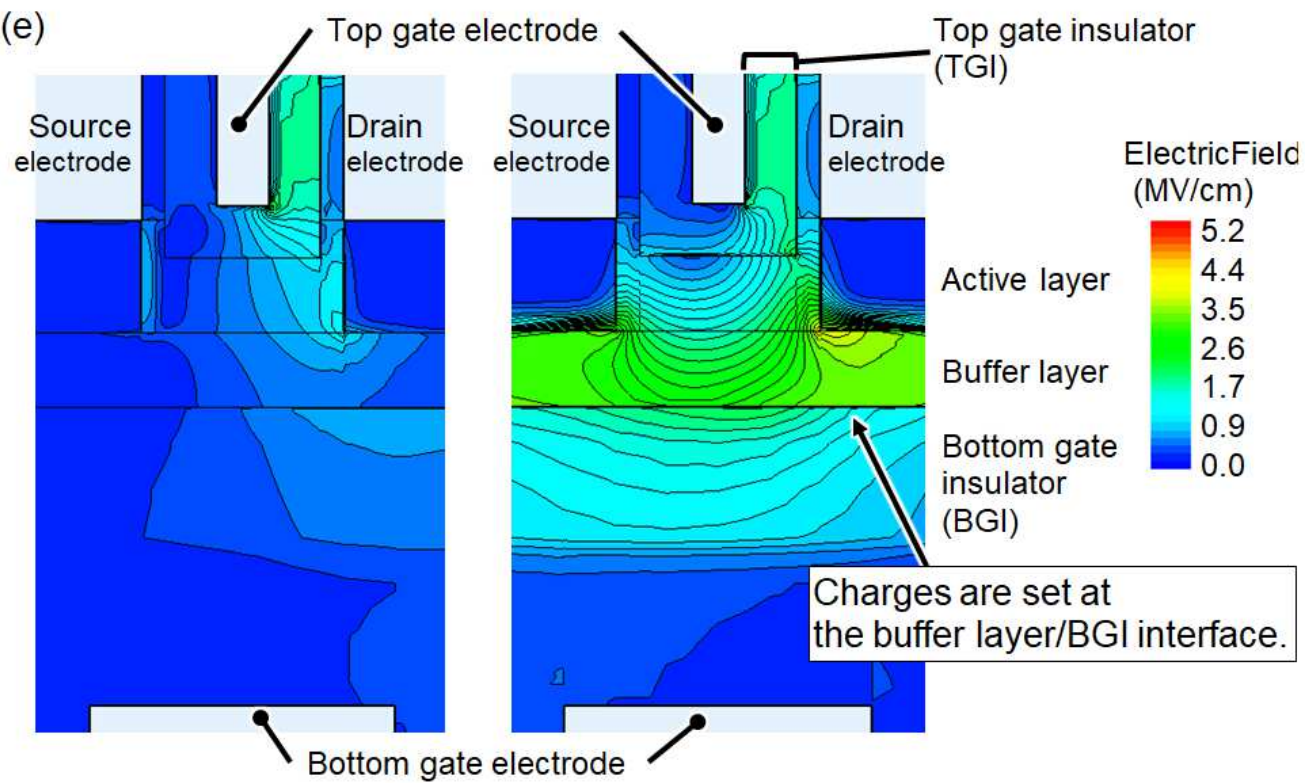

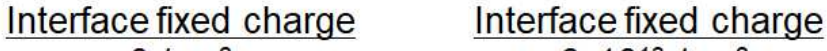
$0 / \mathrm{cm}^{2}$
$-3 \times 10^{13} / \mathrm{cm}^{2}$

Fig. 4 Comparison of results of device simulation in the presence and absence of negative fixed charges $\left(V_{\mathrm{bs}}=\mathbf{0} \mathrm{V}\right)$. (a) $l_{\mathrm{d}}-V_{\mathrm{gs}}$ characteristics $\left(V_{\mathrm{ds}}=0.1 \mathrm{~V}\right.$ or $\left.1.2 \mathrm{~V}\right)$. (b) $S S\left(V_{\mathrm{ds}}=0.1 \mathrm{~V}\right)$. (c) $V_{\mathrm{th}}\left(V_{\mathrm{ds}}=0.1 \mathrm{~V}\right)$. (d) DIBL $\left(V_{\mathrm{ds}}=0.1 \mathrm{~V}\right.$ or $\left.1.2 \mathrm{~V}\right)$. (e) Electric-field intensity distribution $\left(V_{\mathrm{ds}}=1.2 \mathrm{~V}, V_{\mathrm{gs}}=0 \mathrm{~V}\right)$.

interface between an insulating film and a semiconductor, at which electron traps are easily formed, is present only on the top gate side, so that the influence of electron traps during circuit operation can be reduced.

In the second method, negative fixed charges are formed at the interface between the BGI and the buffer layer. In a typical method for controlling the bottom gate-side electric field, the electric field is controlled
399 with the bottom gate electrode or is 400 increased by reducing the thickness of the 401 BGl. We have already attempted control of 402 the electric field by formation of a bottom 403 gate electrode. However, since the BGI is 404 used as not only an insulating film but also a 405 path for diffusion of oxygen to a wide range, 406 a reduction in the BGI thickness is desired to 407 be avoided. To overcome the insufficiency of 408 the bottom gate-side electric field control 
(a)

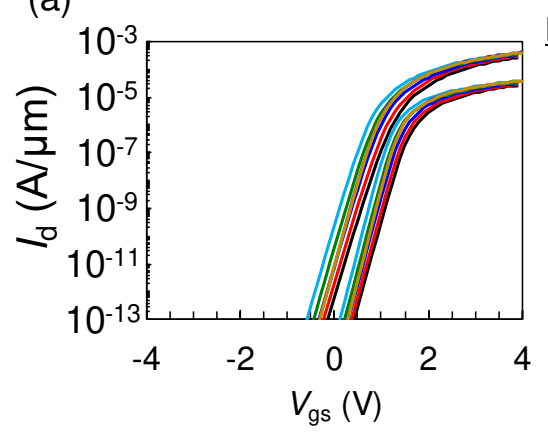

Bottom gate Setting

$-\mathrm{Vbs}=-4 \mathrm{~V}$

$-\mathrm{Vbs}=-2 \mathrm{~V}$

$-\mathrm{Vbs}=0 \mathrm{~V}$

$-\mathrm{Vbs}=+2 \mathrm{~V}$

$-\mathrm{Vbs}=+4 \mathrm{~V}$

-Dual gate drive

(c)

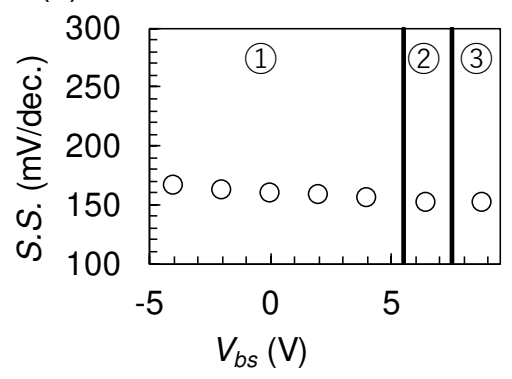

(1) Fixed $V_{\text {bs }}$

(2) Dual gate drive

(3) GAA structure
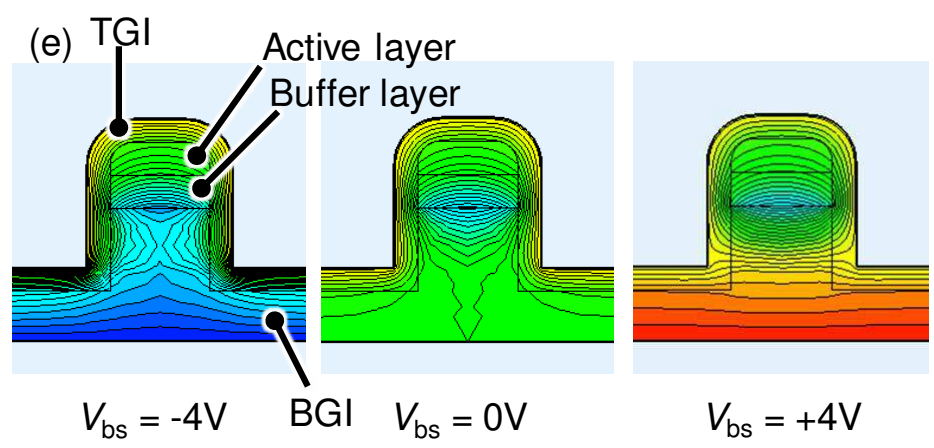

(b)

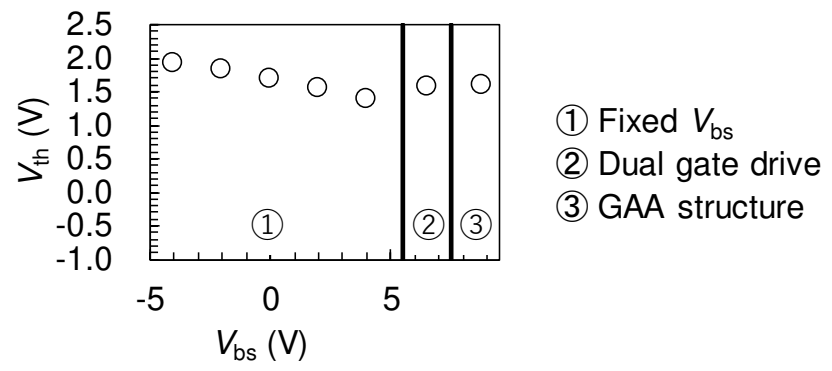

(d) $\mathrm{DIBL}=\frac{V_{\text {th }}\left(V_{\text {ds }}=0.1 \mathrm{~V}\right)-V_{\text {th }}\left(V_{\text {ds }}=1.2 \mathrm{~V}\right)}{\Delta V_{\text {ds }}}$

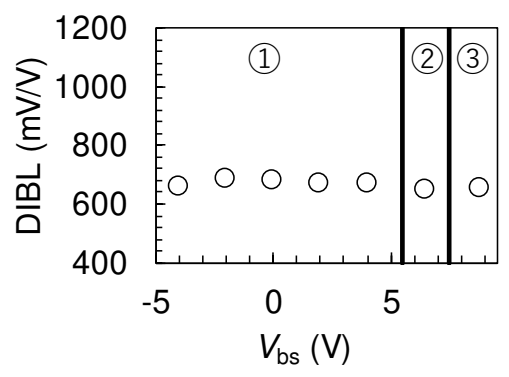

(1) Fixed $V_{b s}$

(2) Dual gate drive

(3) GAA structure

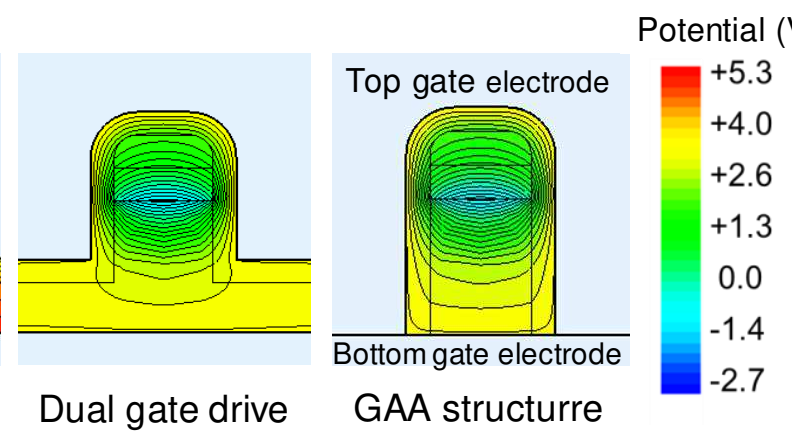

Fig. 5 | Results of device simulation with various bottom gate settings $\left(V_{b s}=0 \mathrm{~V}\right)$. (a) $l_{d}-V_{g s}$ characteristics $\left(V_{\mathrm{ds}}=0.1 \mathrm{~V}\right.$ or $\left.1.2 \mathrm{~V}\right)$. (b) $S S\left(V_{\mathrm{ds}}=0.1 \mathrm{~V}\right)$. (c) $V_{\text {th }}\left(V_{\mathrm{ds}}=0.1 \mathrm{~V}\right)$. (d) DIBL $\left(V_{\mathrm{ds}}=0.1 \mathrm{~V}\right.$ or $\left.1.2 \mathrm{~V}\right)$. (e) Distribution of potential in the $W$ direction $\left(V_{\mathrm{gs}}=V_{\mathrm{th}}+1 \mathrm{~V}, V_{\mathrm{ds}}=1.2 \mathrm{~V}\right)$.

with the existing film structure, we examined the method in which negative fixed charges are formed at the interface between the BGI and the buffer layer. Figs. 4(a) to 4(d) show comparison of the $l_{d}-V_{\mathrm{gs}}$ characteristics, $S S$, $V_{\text {th }}$, and DIBL under negative fixed charge conditions. In calculation of $V_{\text {th }}$, a linear extrapolation method was employed. By setting negative fixed charges, the $l_{\mathrm{d}}-V_{\mathrm{gs}}$ curve was considerably shifted positively, the SS was improved, and DIBL was substantially saturated. In other words, the controllability by the gate electrode was enhanced and the influence of DIBL was suppressed by setting negative fixed charges. Fig. 4(e) shows electric-field intensity distribution in the presence and absence of negative fixed charges. As
427 shown in the diagram, the presence of 428 negative fixed charges blocked the bottom429 gate side drain electric field. It is thus 430 possible to block the bottom-gate side drain 431 electric field by formation of negative fixed 432 charges despite a thick BGI, enabling 433 fabrication of a device that hardly suffers 434 from short channel effect. In addition, the $V_{\text {th }}$ 435 of our CAAC-IGZO FET can be shifted by 436 application of freely set voltage to the bottom 437 gate electrode. The CAAC-IGZO FET can 438 also work like an FET having a GAA 439 structure when the potential of the bottom 440 gate electrode is synchronized with that of 441 the top gate electrode. Fig. 5(a) shows 442 comparison of $l_{d}-V_{g s}$ characteristics with the $443 V_{\text {bs }}$ application mode varied. In the GAA 444 structure, the insulating film between the top 
and bottom gate electrodes was etched around the FET to make the top gate electrode come into direct contact with the bottom gate electrode. Under application of constant voltage, $V_{\text {th }}$ depended on the $V_{\text {bs }}$ as shown in Fig. 5(b). The SS improved in dual gate driving but was not very different from that in the GAA structure (Fig. 5(c)). DIBL was not substantially different between the conditions (Fig. 5(d)). Accordingly, in our CAAC-IGZO FET, the operation mode can be switched to fit the purpose by changing the setting of the bottom gate electrode. Fabrication of this CAAC-IGZO FET in which short channel effect is suppressed will be described in the next section.

\section{Prototype Fabrication and Evaluation}

Figs. 6(a) and 6(b) show cross-sectional STEM images of a CAAC-IGZO FET we prototyped. The cross-sectional images show that our CAAC-IGZO FET has a GAAlike structure in which three surfaces are covered with the top gate and the other surface is covered with the bottom gate and that a fine shape with an $L_{g}$ of $6.8 \mathrm{~nm}$ and a $W$ of $32.1 \mathrm{~nm}$ was formed. For higher integration, not only an FET but also lead wiring and other components therearound must be miniaturized. As shown in the planview image in Fig. 6(c), the prototype achieved a density as high as $127 \mathrm{Tr} / \mu \mathrm{m}^{2}$. The IGZO layer as the active layer was confirmed to have a CAAC structure. Figs. 6(d) and 6(e) show the $l_{d}-V_{g s}$ characteristics of our CAAC-IGZO FET. As shown in Fig. $6(\mathrm{~d}), \quad V_{\text {th }}=1.86 \mathrm{~V}$ $(\sigma=0.169 \mathrm{~V}), \quad S S=172 \mathrm{mV} / \mathrm{dec}$. $(\sigma=18 \mathrm{mV} / \mathrm{dec}),$.$\quad and \quad g_{\mathrm{m}}=66 \mu \mathrm{S} / \mu \mathrm{m}$ $(\sigma=10 \mu \mathrm{S} / \mu \mathrm{m})$ when the drain source voltage $\left(V_{d s}\right)$ was $1.2 \mathrm{~V}$, and the DIBL coefficient between $V_{\mathrm{ds}}=0.1 \mathrm{~V}$ and $1.2 \mathrm{~V}$ was $370 \mathrm{mV} / \mathrm{V} \quad(\sigma=134 \mathrm{mV} / \mathrm{V})$. As described above, an adequate on/off ratio was obtained and normally-off characteristics where $V_{\text {th }}>0 \mathrm{~V}$ were exhibited, even with $L_{g}<10 \mathrm{~nm}$. Moreover,
492 the off-state leakage current was below the 493 lower detection limit despite the $L_{g}$ smaller 494 than $10 \mathrm{~nm}$, and background noise 495 originating from the measurement 496 equipment was observed in the off region. 497 Fig. 6(e) shows the $l_{d}-V_{g s}$ characteristics 498 when the $V_{\text {bs }}$ was increased from $-4 \mathrm{~V}$ to $499+4 \mathrm{~V}$ in steps of $1 \mathrm{~V}$. The $l_{d}-V_{g s}$ curve 500 showed a parallel shift at almost equal 501 intervals as the $V_{\text {bs }}$ was increased, indicating 502 that only the $V_{\text {th }}$ changed. Our CAAC-IGZO 503 FET has a Fin FET-like structure provided 504 with a top gate electrode and a bottom gate 505 electrode. Our CAAC-IGZO FET can have a 506 geometry resembling a GAA structure when 507 in-phase signals are applied to the top gate 508 and the bottom gate, and can make the 509 gates function differently such that a control 510 signal is input from the top gate and $V_{\text {th }}$ is 511 controlled by the bottom gate as shown in 512 Fig. 6(e) when power is independently 513 supplied to the bottom gate.

514 To examine the performance in a logic circuit, 515 a ring oscillator serving as an oscillator 516 circuit was prototyped by serial connection of 517 an odd number of inverters in each of which 518 an n-channel CAAC-IGZO FET was stacked 519 monolithically over a Si p-channel metal520 oxide semiconductor (PMOS) circuit 521 (Fig. 6(f)). The oscillation frequency and 522 delay time of the ring oscillator were 523 evaluated. Fig. 6(g) schematically shows the 524 3D layout of the ring oscillator circuit with an 525 IGZO/Si monolithic stack. This circuit was 526 fabricated by monolithically stacking CAAC527 IGZO FETs over SiFETs formed in a front528 end-of-line (FEOL) process, as shown in the 529 cross-sectional image in Fig. 6(h). In the 530 evaluation, an output signal $V_{\text {osc }}$ of a 51531 stage ring oscillator was observed with an 532 oscilloscope. When in-phase signals were 533 applied to the top and bottom gates of the 534 CAAC-IGZO FET, the oscillation frequency 535 was $9.1 \mathrm{MHz}$ as shown in Fig. 6(i), which 536 can be converted into a delay time per stage 537 of $1.1 \mathrm{~ns}$. Fig. 6(j) shows examination results 538 of $V_{\text {th }}$ control by the bottom gate in the 

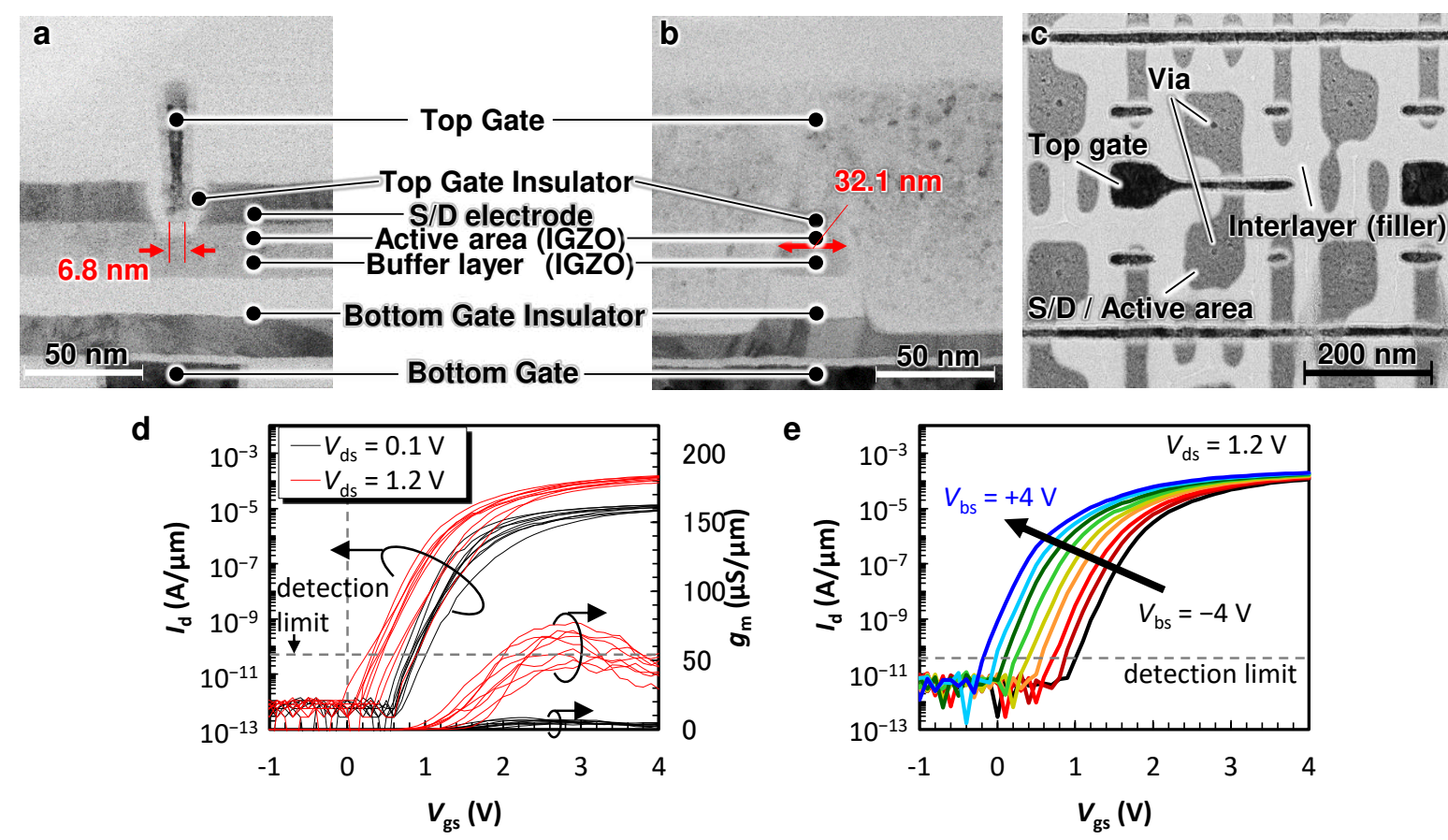

f

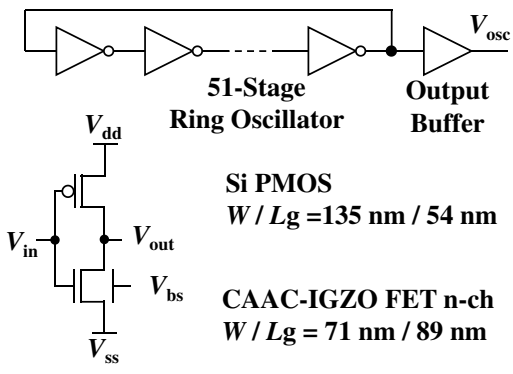

g

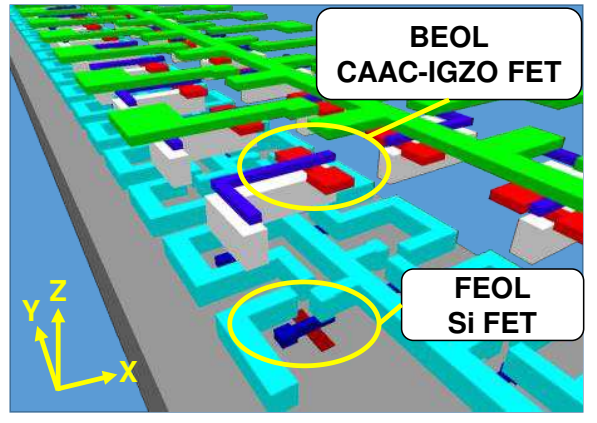

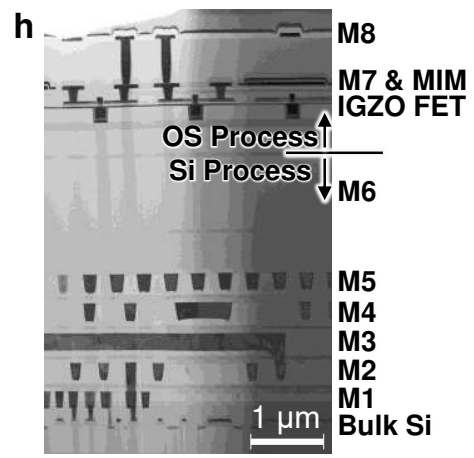

$\mathbf{k}$

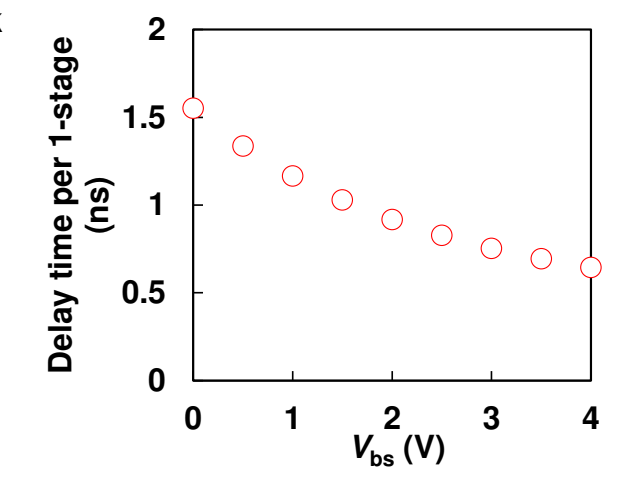

Fig. 6 | Fabricated CAAC-IGZO FET and evaluation results of circuit. (a), (b) Cross-sectional STEM images of our CAAC-IGZO FET ( $W / L_{g}=20 \mathrm{~nm} / 6.8 \mathrm{~nm}$ ) in the Lch direction (a) and the $W$ direction (b). (c) A plan-view STEM image of a CAAC-IGZO FET array $\left(W / L_{g}=33.1 \mathrm{~nm} / 19.2 \mathrm{~nm}\right)$ having a density of $127 \mathrm{Tr} / \mu \mathrm{m}^{2}$. (d), (e) Transfer characteristics of CAAC-IGZO FETs (W/L $=20 \mathrm{~nm} / 20 \mathrm{~nm}$ ) as a function of $V_{\mathrm{ds}}(\mathrm{d})$ and $V_{\mathrm{bs}}(\mathrm{e})$. (f) A circuit diagram of a ring oscillator fabricated by monolithically stacking n-channel CAAC-IGZO FETs over a Si PMOS circuit. (g) A 3D image of an actual layout of the ring oscillator. (h) A cross-sectional STEM image of the ring oscillator. (i), (j) Output waveforms of the ring oscillator. (k) Delay time of the ring oscillator as a function of $V_{\text {bs. }}$.

i

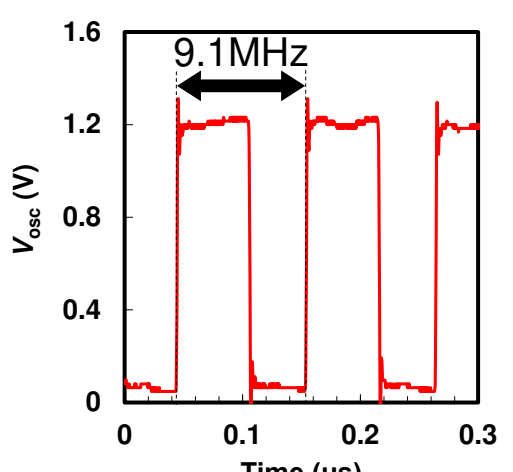

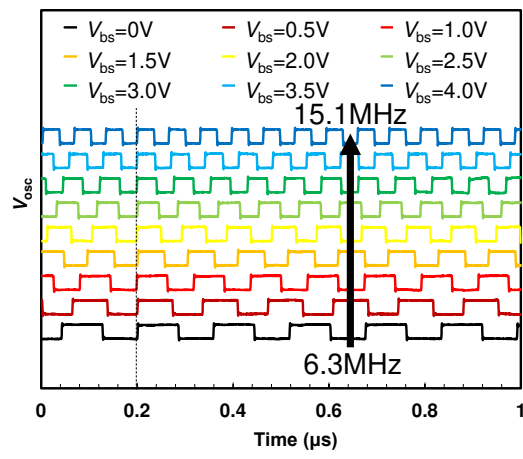

542 As a result of $V_{\text {bs }}$ application from $0 \mathrm{~V}$ to $4 \mathrm{~V}$, 543 the oscillation frequency was found to 544 increase approximately 2.4 times and the 
delay time per stage was shortened to approximately $0.6 \mathrm{~ns}$. As described above, CAAC-IGZO FETs can be fabricated in a BEOL process and can also be used for a logic circuit combined with a Si PMOS circuit.

\section{CONCLUSION}

A miniaturized TGSA FET having a Fin-type top gate electrode and a bottom gate electrode and including CAAC-IGZO in the channel layer was fabricated. We developed a method for forming a junctionless transistor with the $n^{-}$type channel and the $n^{+}$ type source and drain regions by appropriately controlling $V_{0}$, hydrogen concentration, and band alignment around CAAC-IGZO during the CAAC-IGZO FET fabrication process. The CAAC-IGZO FET formed by this process exhibited excellent normally-off characteristics with a $V_{\text {th }}$ of $1.86 \mathrm{~V}$ and an $S S$ of $172 \mathrm{mV} /$ dec despite the $L_{g}$ as small as $6.8 \mathrm{~nm}$. We also prototyped a ring oscillator composed of a Si PMOS circuit and CAAC-IGZO FETs formed in a BEOL process, to find out that the ring oscillator operated at an oscillation frequency of $9.1 \mathrm{MHz}$ and with a delay time per stage of $1.1 \mathrm{~ns}$. A junctionless IGZO FETs in which short channel effect is inhibited by the above improvements is expected to be applied to a low-power memory cell array, a selector of a leadingedge embedded memory, an analog amplifier circuit, a high speed logic circuit, and other devices is expected.

\section{EXPERIMENTAL METHOD}

\section{Device simulation}

In the calculation of the device characteristics by device simulation, Sentaurus Device manufactured by Synopsys G.K. was used. Other than the varied conditions, the simulation employed the following parameters in principle. For the active layer, the electron mobility was $6 \mathrm{~cm}^{2} / \mathrm{Vs}$, the hole mobility was $0.01 \mathrm{~cm}^{2} / \mathrm{Vs}$,
591 the band gap was $3.2 \mathrm{eV}$, the electron 592 affinity was $4.7 \mathrm{eV}$, the donor concentration 593 of the channel and LDD regions was $59410^{16} / \mathrm{cm}^{3}$, and the donor concentration of the 595 source and drain regions was $10^{20} / \mathrm{cm}^{3}$. For 596 the buffer layer, the electron mobility was $5971.5 \mathrm{~cm}^{2} / \mathrm{Vs}$, the hole mobility was $5980.01 \mathrm{~cm}^{2} / \mathrm{Vs}$, the band gap was $3.4 \mathrm{eV}$, the 599 electron affinity was $4.5 \mathrm{eV}$, the donor 600 concentration of the channel and LDD 601 regions was $10^{10} / \mathrm{cm}^{3}$, and the donor 602 concentration of the source and drain 603 regions was $10^{10} / \mathrm{cm}^{3}$. In each layer, the 604 dielectric constant was 15 and the density of 605 state in the conduction band and that in the 606 valence band were $5 \times 10^{18} / \mathrm{cm}^{3}$. The 607 thickness of the TGl was $7 \mathrm{~nm}$ and the 608 dielectric constant thereof was 5.5. The 609 thickness of the BGI was $40 \mathrm{~nm}$ and the 610 dielectric constant thereof was 6.6. The work 611 functions of the top gate electrode, the 612 bottom gate electrode, and the source and 613 drain electrodes were $4.7 \mathrm{eV}, 5.0 \mathrm{eV}$, and $6144.5 \mathrm{eV}$, respectively. The trench gate had a 615 width of $21 \mathrm{~nm}$, and its length was $7 \mathrm{~nm}$ 616 owing to the TGI formed on both side walls. 617 The Fin width was $30 \mathrm{~nm}$, which was defined 618 as the W.

619 Fabrication of IGZO thin film and 620 characterization

621 The IGZO thin films having the compositions 622 A, B, C, and D shown in Figs. 2(c) and 1(d) 623 were formed using IGZO targets having 624 different In: Ga: Zn ratios. All the films were 625 deposited using a radio-frequency 626 magnetron sputtering apparatus, although 627 the film having the composition $B$ was 628 formed using a deposition apparatus 629 different from that used for the other films. In 630 the phase diagram in Fig. 2(c), the 631 compositions $A, B, C$, and $D$ are not the 632 compositions of the targets but the 633 compositions of the 100-nm-thick IGZO thin 634 films each formed on a Si substrate, which 635 were examined by inductively coupled 636 plasma mass spectrometry using an Agilent 637 Technologies, Inc. Agilent 8900. The optical 638 band gaps shown in Fig. 2(d) were each 
639 calculated by creating a Tauc plot using the 640 optical absorption coefficient of the IGZO 641 thin film obtained by spectroscopic 642 ellipsometry using a HORIBA, Ltd. UT-300. 643 The carrier concentrations in Fig. 2(e) were 644 each obtained by performing Hall effect 645 measurement by the van der Pauw method 646 on a 35-nm-thick CAAC-IGZO thin film which was formed on a quartz substrate and annealed at $450^{\circ} \mathrm{C}$ in an oxygen atmosphere for 1 hour. The Hall effect measurement was performed using a TOYO Corporation ResiTest 8400 . Note that the results in Fig. 2(e) were obtained in such a manner that the carrier concentration was increased by deliberately forming $\mathrm{Vo}$ and $\mathrm{VoH}$ by annealing on a deposited CAAC-IGZO thin film at $400^{\circ} \mathrm{C}$ under vacuum for 1 hour and subsequently, annealing was performed at $200^{\circ} \mathrm{C}, 250^{\circ} \mathrm{C}, 300^{\circ} \mathrm{C}, 350^{\circ} \mathrm{C}$, or $400^{\circ} \mathrm{C}$ in an oxygen atmosphere for 1 hour.

Fabrication of IGZO FET and IGZO FETS/SiFETs monolithic integration

The SiFETs were formed by a $55-\mathrm{nm}$ process. Over the thus obtained Si wafer, a 400-nm-thick planarization film of $\mathrm{SiO}_{2}$ was formed, followed by contact formation. Then, a wiring layer serving as both a bottom gate electrode and an interconnect to the Si wafer was formed. The BGI was a combination of 20-nm-thick $\mathrm{HfO}_{2}$ and 20-nm-thick $\mathrm{SiO}_{2}$. Next, source/drain electrode patterns except for the active layer and the channel region were formed at a time. After formation of 10nm-thick IGZO and 15-nm-thick IGZO having different compositions, baking was performed at $450^{\circ} \mathrm{C}$ under atmospheric pressure for 1 hour, and 20-nm-thick TaN was deposited to form source and drain electrodes. Then, the channel region and gate electrode were formed by a damascene process in a self-aligned manner. The $\mathrm{Gl}$ was a combination of 1-nm-thick $\mathrm{Al}_{2} \mathrm{O}_{3}$, 3nm-thick $\mathrm{SiO}_{2}$, and 3-nm-thick $\mathrm{Si}_{3} \mathrm{~N}_{4}$. The gate electrode over and in direct contact with the channel was a combination of 5-nm-thick TiN and 75-nm-thick $W$ each deposited by metal chemical vapor deposition.
687 Photolithographic patterning was adopted in 688 forming contact with the Si wafer.

\section{Measurement}

690 The transfer characteristics were evaluated 691 using a semiautomatic prober manufactured 692 by HiSOL, Inc. at a stage temperature of $69327^{\circ} \mathrm{C}$ in a dry air under atmospheric pressure. 694 As measurement equipment, a Keysight 695 Technologies B1500A was used. $V_{d s}$ was $6960.1 \mathrm{~V}$ or $1.2 \mathrm{~V}$ and $V_{\text {bs }}$ was $0 \mathrm{~V}$. The device 697 characteristics parameters described in 698 Section IV are the medians of nine samples. 699 Furthermore, $V_{\text {th }}$ was calculated by a linear 700 extrapolation method.

701 The ring oscillator was evaluated at a 702 stage temperature of $27^{\circ} \mathrm{C}$ in an indoor 703 environment (atmospheric pressure, 704 humidity: 50\%) using a semiautomatic 705 prober manufactured by HiSOL, Inc. As 706 measurement equipment, a Keysight 707 Technologies B1500A was used, and $V_{D D}$ 708 and $V_{\text {Ss }}$ were $1.2 \mathrm{~V}$ and $0 \mathrm{~V}$, respectively. 709 The waveform was read out with the use of 710 a Model 28 Picoprobe $^{\circledR}$ manufactured by 711 GGB Industries, Inc. and a GDS-3504 GW 712 Instek oscilloscope so that the output load 713 can be minimized.
714

715

716

717

718

719

720

721

722

723

724

725

726

727

728

729

730

731

732

733

\section{REFERENCES}

[1] K. Zhao, "Beyond FINFET era Challenges and Opportunities for CMOS Technology," Symposium on IEDM, Tutorial-1, 2021.

[2] M. Tada, "CMOS Technology Enablers for Pushing the Limits of Semiconductors: Materials to Packaging," Symposium on VLSI Technology and Circuits, Short Course 1, 2019.

[3] P. Batude, "3D sequential integration," Symposium on IEDM, Tutorial-6, pp. 7-11, 2019.

[4] S. Frank, "Nanometer CMOS. Pan Stanford Publishing," pp. 17, 2010.

[5] "International Roadmap for Devices and Systems (IRDS) 2021 Edition"

[6] "International Technology Roadmap for Semiconductors (ITRS) 2001 Edition"

[7] W. Li et al., "High-Performance CVD MoS2 Transistors with Self-Aligned Top-Gate and 
Bi Contact," IEDM Tech. Dig., pp. 37.3.137.3.4, 2021. (10.1021/nl303583v)

[8] C. D. English et al., "Approaching Ballistic Transport in Monolayer MoS2 Transistors with Self-Aligned $10 \mathrm{~nm}$ Top Gates," IEDM Tech. Dig., pp. 5-6, 2016. (10.1109/IEDM.2016.7838355)

[9] H. Watakabe et al., "Development of Advanced LTPS TFT Technology for Low Power Consumption and Narrow Border LCDs," SID Symp. Dig. Tech. Pap., vol. 50, Issue 1, pp. 541-544, 2019. (10.1002/sdtp.12977)

[10] R. Yonebayashi et al., "High refresh rate and low power consumption AMOLED panel using top-gate n-oxide and p-LTPS TFTs," J. Soc. Info. Disp., vol. 28, Issue 4, pp. 350359, 2020. (10.1002/jsid.888)

[11] U.-J. Chung et al., "Manufacturing Technology of LTPO TFT," SID Symp. Dig. Tech. Pap., vol. 50, Issue 1, pp. 192-195, 2020. (10.1002/sdtp.13835)

[12] S. Yamazaki et al., "Crystalline IGZO ceramics (crystalline oxide semiconductor) based devices for artificial intelligence," International Journal of Ceramic Engineering \& Science, vol. 1, pp. 6-20, 2019. (10.1002/ces2.10005)

[13] S. Yamazaki et al., "Research, Development, and Application of Crystalline Oxide Semiconductor," SID Symp. Dig. Tech. Pap., pp. 183-186, 2012. (10.1002/j.2168-0159.2012.tb05742.x)

[14] M. Takahashi et al., "C-Axis Aligned Crystalline In-Ga-Zn-Oxide FET with High Reliability," Proc. AM FPD'11, pp. 271-274, 2011.

[15] R. Honda et al., "Stress Durability of CAACIGZO TFT," IDW'15 Proc., pp. 270-273, 2015.

[16] Y. Kobayashi et al., "Scaling to 100nm Channel Length of Crystalline In-Ga-ZnOxide Thin Film Transistors with Extremely Low Off-State Current," Ext. Abstr. SSDM, pp. 930-931, 2013.

[17] T. Onuki et al., "Display and LSI Applications of Oxide Semiconductor LSIs (OSLSIs) Using Crystalline In-Ga-Zn Oxide (IGZO): Applications Related to Coronavirus COVID-19 Pandemic," 2020 ECS Trans., vol. 98, pp. 185-204, 2020. (10.1149/09807.0185ecst)

[18] S. Samanta et al., "Amorphous IGZO TFTs featuring Extremely-Scaled Channel
788

789

790

791

792

793

794

795

796

797

798

799

800

801

802

803

804

805

806

807

808

809

810

811

812

813

814

815

816

817

818

819

820

821

822

823

824

825

826

827

828

829

830

831

832

833

834

835

836

837

838

839

840

841
Thickness and $38 \mathrm{~nm}$ Channel Length: Achieving Record High Gm max of 125 $\mu \mathrm{S} / \mu \mathrm{m}$ at VDS of $1 \mathrm{~V}$ and ION of 350 $\mu \mathrm{A} / \mu \mathrm{m}, "$ Symp. on VLSI Tech. Dig., pp. 1-2, 2020.

(10.1109/VLSITechnology18217.2020.9265 052)

[19] H. Kunitake et al., "High thermal tolerance of 25-nm c-axis aligned crystalline In-Ga-Zn oxide FET," IEDM Tech. Dig., pp. 13.6.113.6.4, 2018. (10.1109/IEDM.2018.8614621)

[20] S. Subhechha et al., "First demonstration of sub-12 nm Lg gate last IGZO-TFTs with oxygen tunnel architecture for front gate devices," Symp. on VLSI Tech. Dig., pp. T10-5, 2021.

[21] A. Suzuki et al., "Characteristics and Applications of CAAC-IGZO FET with Gate Length of $13 \mathrm{~nm}, "$ ECS Transactions, vol. 98, pp. 13-17, 2020. (10.1149/MA2020$02281914 \mathrm{mtgabs})$

[22] John Robertson, "High dielectric constant gate oxides for metal oxide Si transistors," Rep. Prog. Phys., vol. 69, pp. 327-396, 2005. (10.1088/0034-4885/69/2/R02)

[23] S. H. Wu et al., "Extremely Low Power CAxis Aligned Crystalline In-Ga-Zn-O $60 \mathrm{~nm}$ Transistor," Symp. on VLSI Tech. Dig., pp. 58-59, 2016. (10.1109/VLSIT.2016.7573378)

[24] N. Okuno et al., "Source/Drain Engineering by Tantalum Nitride (TaNx) Electrode for Boosting OSFET Performance," IEDM Tech. Dig., pp. 37.5.1-37.5.4, 2021.

[25] M. Nakashima et al., "Origin of major donor states in In-Ga-Zn oxide," J. Appl. Phys., vol. 116, pp. 213703-1 - 213703-9, 2014. (10.1063/1.4902859)

[26] Y. Asami et al., "Properties of c-axis-aligned crystalline indium-gallium-zinc oxide fieldeffect transistors fabricated through a tapered-trench gate process," Jpn. J. Appl. Phys., vol. 55, pp. 04EG09, 2016. (10.7567/JJAP.55.04EG09)

[27] D. Matsubayashi et al., "20-nm-Node trench-gate-self-aligned crystalline In-Ga$\mathrm{Zn}$-Oxide FET with high frequency and low off-state current," IEDM Tech. Dig., pp. 141, 2015. (10.1109/IEDM.2015.7409641)

[28] Y. Kobayashi et al., "Electrical characteristics and short-channel effect of caxis aligned crystal indium gallium zinc oxide transistor with short channel length," 
Jpn. J. Appl. Phys., vol. 53, pp. 04EF03, 2014. (10.7567/JJAP.53.04EF03)

[29] F. D'Agostino et al., "Short-Channel Effects in MOSFETs," Introduction to VLSI design, 2020., Available: http://www0.cs.ucl.ac.uk/staff/ucacdxa/proj ects/vlsi/report.pdf

[30] E. Fortunato et al., " Oxide Semiconductor Thin-Film Transistors: A Review of Recent Advances" ADVANCED MATERIALS, vol.24, pp. 2945-2986, 2012. (10.1002/adma.201103228)

[31] H. Kunitake et al., "Crystalline Oxide Semiconductor Applicable to Low-Power Consumption Edge Al," ECS Transactions, vol. 102(2), pp. 3-18, 2021. (10.1149/10202.0003ecst)

[32] D. Matsubayashi et al., "Conduction Band Lowering Effect in Crystalline IndiumGallium-Zinc-Oxide Thin Film Transistors," ECS Transactions, vol. 54(1), pp. 115-120, 2013. (10.1149/05401.0115ecst)

[33] K. Kato et al., "Evaluation of Off-State Current Characteristics of Transistor Using Oxide Semiconductor Material, IndiumGallium-Zinc Oxide," Jpn. J. Appl. Phys. vol. 51, pp. $\quad 021201,2012$. (10.1143/JJAP.51.021201)

[34] H. Kunitake et al., "A c -Axis-Aligned Crystalline In-Ga-Zn Oxide FET with a Gate Length of $21 \mathrm{~nm}$ Suitable for Memory Applications," IEEE Journal of the Electron Devices Society, vol. 7, pp. 495 - 502, 2019. (10.1109/JEDS.2019.2909751)

[35] T. Ono et al., "Hydrogen Absorption Method Using HfOx in Crystalline In-Ga-Zn Oxide FETs for NVM Applications," IEDM Tech. Dig., $\quad$ pp. $\quad 39.4 .1-39.4 .4,2020$. (10.1109/IEDM13553.2020.9372030)

[36] M. Kaneyasu et al., "New Pixel Circuits for Controlling Threshold Voltage by Back-gate Bias Voltage using Crystalline Oxide Semiconductor FETs," SID Symp. Dig. Tech. Pap., $\quad$ pp. 857-860, 2015. (10.1002/sdtp.10363)

[37] Bin Yu et al., "FinFET Scaling to 10nm Gate Length," IEDM Tech. Dig., pp. 251-254, 2002. (10.1109/IEDM.2002.1175825)

[38] K. Tsutsui et al., "Mobility enhancement in crystalline In-Ga-Zn-oxide with In-rich composition," Appl. Phys. Lett., vol. 107, pp. 262104, 2015. (10.1063/1.4939039) 


\section{Supplementary Files}

This is a list of supplementary files associated with this preprint. Click to download.

- NATELECTRON21124815supplyfile.docx 